\title{
ON PROPERTIES OF SELF RECIPROCAL FUNCTIONS
}

BY V. V. L. N. RAO

Communicated by M. Gerstenhaber, March 25, 1968

Following is the notation of Hardy and Titchmarsh [1]. We denote a function as $R \mu$ if it is self reciprocal for Hankel transforms of order $\mu$, so that it is given by the formula

$$
f(x)=\int_{0}^{\infty} J_{\mu}(x y) f(y) \sqrt{x y} d y,
$$

where $J_{\mu}(x)$ is a Bessel function of order $\mu$. For $\mu=\frac{1}{2}$ and $-\frac{1}{2}, f(x)$ is denoted as $R_{\mathrm{s}}$ and $R_{\mathrm{c}}$ respectively.

Brij Mohan [2] has shown that if $f(x)$ is $R \mu$, and

$$
P(x)=\frac{1}{2 \pi i} \int_{c-i \infty}^{c+i \infty} 2^{s} \Gamma\left(\frac{1}{4}+\frac{\mu}{2}+\frac{s}{2}\right) \Gamma\left(\frac{1}{4}+\frac{\nu}{2}+\frac{s}{2}\right) \theta(s) x^{-s} d s,
$$

where

$$
\theta(s)=\theta(1-s) \text { and } 0<c<1,
$$

then $P(x)$ is a Kernel transforming $R_{\mu}\left(R_{\nu}\right)$ into $R_{\nu}\left(R_{\mu}\right)$. As an example of (1.2) Brij Mohan has shown that the function

$$
x^{y+1 / 2} e^{-x}
$$

is a Kernel transforming $R_{v}$ into $R_{p+1}$. In particular, putting $\nu=\frac{1}{2}$, we find that the Kernel

$$
x e^{-x},
$$

transforms $R_{8}$ into $R_{3 / 2}$. Again, I have shown in a previous paper [3] that the Kernel

$$
\sqrt{x} e^{-x / 2}
$$

transforms $R_{1}$ into $R_{2}$. From (1.4) and (1.5) we find that "A Kernel transforming $R_{1}$ into $R_{2}$ will have its square transforming $R_{\mathbf{t}}$ into $R_{3 / 2}$." Again Sneddon [4] has shown that

$$
\int_{0}^{\infty} e^{-x} x^{m} \operatorname{Ln}(x) d n=(-1)^{m} m ! \int_{0}^{\infty} \frac{d^{n-m}}{d n^{n-m}}\left(x^{n} e^{-x}\right) d x,
$$

$\operatorname{Ln}(x)$ being Laguerre polynomial of order $n$. Putting $m=n$, we obtain that 


$$
\int_{0}^{\infty} e^{-x} x^{n} \operatorname{Ln}(x) d x=(-1)^{n} n ! \int_{0}^{\infty} x^{n} e^{-x} d x .
$$

On writing $x^{2}$ for $x$, we find that

$$
\int_{0}^{\infty} e^{-x^{2}} x^{2 n+1} \operatorname{Ln}\left(x^{2}\right) d x=(-1)^{n} n ! \int_{0}^{\infty} x^{2 n+1} e^{-x^{2}} d x .
$$

Brij Mohan [5] has shown that the function

$$
e^{-x^{2} / 2} x^{2 n+1 / 2}
$$

is $R_{2 n}$; while Howell [6] has shown that the function

$$
e^{-x^{2} / 2} x^{1 / 2} \operatorname{Ln}\left(x^{2}\right),
$$

is $R_{0}$.

Hence, from (1.8) and (1.9) we find that the integral on the lefthand side of (1.7) is the product of the functions which are $R_{0}$ and $R_{2 n}$. Again, by writing the integrand on the right-hand side of (1.7) in the form

$$
x^{n} e^{-x^{2} / 2} n^{n+1} e^{-x^{2} / 2},
$$

we find from (1.8) that it is a product of two functions which are $R_{(n-1 / 2)}$ and $R_{(n+1 / 2)}$. Applying the results of (1.8), (1.9) and (1.10) to (1.7), we conclude that the integral of a product of two functions which are $R_{0}$ and $R_{2 n}=$ the integral of a product of two functions which are $R_{n-1 / 2}$ and $R_{n+1 / 2}$. This may be compared with a theorem given by me in a previous paper [7].

Again, the integrand on the right-hand side of (1.7) may also be written in the form

$$
\left(x^{n+1 / 2} e^{-x^{2} / 2}\right)^{2},
$$

which is a square of an $R_{n}$ function. Hence from (1.8), (1.9), (1.11) and (1.7), we further conclude that the integral of a square of an $R_{n}$ function $=$ the integral of the product of two functions which are $R_{0}$ and $R_{2 n}$. My thanks are due to Dr. Brij Mohan for his constant guidance in my research work.

\section{REFERENCES}

1. G. H. Hardy and E. C. Titchmarsh, Self reciprocal functions, Quart. J. Math., Oxford Ser. 1 (1930), 196-231.

2. Brij Mohan, $A$ class of kernels, J. Benares Hindu Univ. Silver Jubilee Number (1942), 134-137.

3. V. V. L. N. Rao, On certain kernel functions, Ganita 9 (1958), 33-41. 
4. I. N. Sneddon, Special functions of mathematical physics and chemistry, Edinburgh (1961), 162.

5. Brij Mohan, Formulae connecting self reciprocal functions, Indian J. Phys. 15 (1941), 337-341.

6. W. T. Howell, On some operational representations, Philos. Mag. 24 (1937), 1082-1083.

7. V. V. L. N. Rao, $A$ property of self reciprocal functions, Vijnana Parishad Anusandhan Patrika (1) 2 (1959), 51-54.

Bholakpur, Secunderabad-3, A. P., India.

\section{REPRESENTATION OF NONLINEAR TRANSFORMATIONS ON $L^{p}$ SPACES}

BY V. J. MIZEL

Communicated by Henry McKean, July 11, 1968

This note describes integral representations obtained for a class of nonlinear functionals and nonlinear transformations on the spaces $L^{p}(T)(1 \leqq p \leqq \infty)$ associated with an arbitrary $\sigma$-finite measure space $(T, \Sigma, \mu)$. The class of functionals considered here differs from those considered in [1], [3], [7], [8], [9] and its study is mainly motivated by its close connection with nonlinear integral equations [6].

In the study of nonlinear integral equations there is a fundamental class of nonlinear transformations, called Urysohn operators [6], taking measurable functions to measurable functions and having the form

$$
(A x)(s)=\int_{T} \phi(s ; x(t), t) d t
$$

where $S, T$ are Lebesgue measurable subsets of $R^{n}$ and $\phi: S \times R \times T \rightarrow R$ is a real valued function which is measurable on $S \times T$ for each fixed value of its second argument and continuous on $R$ for almost all arguments in $S \times T$. An important subclass of (1) consists of those Urysohn operators whose range is in $C(S)$ where $S$ is compact. This subclass includes the case in which the kernel $\phi$ is independent of its first argument, so that the operator reduces to a real valued functional:

$$
F(x)=\int_{\mathbf{T}} \phi(x(t), t) d t .
$$

Functionals of the form (2) also play an important role in the theory of generalized random processes in probability [5]. 